\title{
Internações por condições sensíveis à Atenção Primária à Saúde em crianças menores de 1 ano no Brasil
}

\author{
Primary care-sensitive hospitalization conditions in children under \\ the age of 1 in Brazil
}

Elzo Pereira Pinto Junior (https://orcid.org/0000-0002-6977-2071) ${ }^{1}$

Rosana Aquino (https://orcid.org/0000-0003-3906-5170) ${ }^{1}$

Inês Dourado (https://orcid.org/0000-0003-1675-2146) ${ }^{1}$

Líllian de Queiroz Costa (https://orcid.org/0000-0001-9849-6339) ${ }^{2}$

Marcelo Gurgel Carlos da Silva (https://orcid.org/0000-0003-4030-1206) ${ }^{2}$

${ }^{1}$ Instituto de Saúde Coletiva, Universidade Federal da Bahia. R. Basílio da Gama s/n, Caneta. 40110-040 Salvador BA Brasil.elzojr@ hotmail.com

${ }^{2}$ Programa de Pós-Graduação em Saúde Coletiva,

Universidade Estadual do

Ceará. Fortaleza CE Brasil.

\begin{abstract}
Primary care-sensitive condition hospitalizations (PCSCH) are an essential health care indicator. This ecological, time-series study aimed to analyze the time trend of PCSCH in children under the age of 1 in Brazil, considering the age subcomponents of newborns and post-newborns. The PCSCH rates were calculated for infants under the age of 1 and in the neonatal and postneonatal subgroups. The Prais-Winsten generalized linear analysis model and the Annual Percent Change (APC) calculation were used to evaluate the time trend. The results showed that infectious gastroenteritis represented the most important cause of hospitalizations due PCSCH in children under the age of 1. Congenital syphilis and other congenital infections accounted for the highest proportion of hospitalizations in newborns, whereas gastroenteritis prevailed in post-newborns. An increase in newborn hospitalization rates and a decrease in hospitalization rates in both the postneonatal group and the group of children under the age of 1. Differences in trends in these hospitalization rates may reflect the influence of specific determinants on the risk of hospitalization in each age subcomponent.
\end{abstract}

Key words Primary Health Care, Child Health, Hospitalization, Time-Series Studies, Ecological Studies
Resumo As internações por condições sensiveis à Atenção Primária à Saúde (ICSAP) representam importante indicador do cuidado em saúde. Objetivou-se analisar a tendência temporal das internações por condições sensivivis a APS em menores de 1 ano, considerando os subcomponentes etários dos neonatos e pós-neonatos, no Brasil. Trata-se de um estudo ecológico, de série temporal. Foram calculadas as taxas de ICSAP em menores de 1 ano e nos subgrupos neonatal e pós-neonatal. $O$ modelo de análise linear generalizado de Prais - Winsten e o cálculo da Annual Percente Change (APC) foram utilizados para avaliar a tendência temporal. Os resultados mostraram que as gastroenterites infecciosas representaram a causa mais importante de ICSAP nos menores de 1 ano. Nos neonatos, a sífilis congênita e outras infecções congênitas foram as responsáveis pela maior proporção de internações, enquanto nos pós-neonatos as gastroenterites tiveram maior magnitude. Constatou-se aumento nas taxas de hospitalização dos neonatos e diminuição nas taxas tanto no grupo pós-neonatal quanto no conjunto de menores de 1 ano. As diferenças nas tendências dessas taxas de hospitalizaçôes podem refletir a influência de determinantes específicos no risco de internar em cada um dos subcomponentes etários.

Palavras-chave Atenção Primária à Saúde, Saúde da Criança, Hospitalização, Estudos de Séries Temporais, Estudos Ecológicos 


\section{Introdução}

As condições sensíveis à atenção primária são definidas como um conjunto de doenças e agravos cujas hospitalizações são consideradas evitáveis se as ações desenvolvidas no âmbito da atenção primária à saúde (APS) forem ofertadas oportunamente e tenham caráter resolutivo ${ }^{1}$. No Brasil, foi elaborada uma lista de causas de internações por condições sensíveis à atenção primária (ICSAP), distribuídas em 19 grupos de causas ${ }^{2}$, sendo as gastroenterites infecciosas e suas complicações, as pneumonias bacterianas e a asma os três principais motivos de internamento em crianças menores de 1 ano $^{3}$.

Estudos realizados a partir de dados secundários apontaram um efeito da expansão da Estratégia Saúde da Família (ESF) na redução das ICSAP no Brasil ${ }^{4-6}$. Entretanto, investigações sobre ICSAP em crianças menores de cinco anos conduzidas em diferentes unidades da federação tem apresentado resultados contraditórios. Enquanto em Pernambuco e no Piauí ${ }^{8}$ houve redução nas internações evitáveis, no Paraná ${ }^{9}$ foi observado aumento desses eventos.

Em relação aos menores de 1 ano, ainda são escassos os estudos que analisam a tendência temporal das ICSAP neste grupo etário, especialmente nos seus componentes neonatal e pós-neonatal, no Brasil. No caso da mortalidade infantil, já existem evidências bem estabelecidas que a evolução destas taxas, nas últimas décadas, é marcadamente diferentes nos dois componentes, com lenta redução da mortalidade neonatal, diretamente associada à fatores relacionadas à assistência direta à gravidez, parto e puerpério, e redução em ritmo mais acelerado da mortalidade pós-neonatal, que guarda estreita relação com determinantes sociais da saúde ${ }^{10}$.

Este estudo objetivou analisar a tendência temporal das internações por condições sensíveis a APS em menores de 1 ano, considerando os subcomponentes etários neonatal e pós-neonatal, no Brasil. Nesse sentido, espera-se contribuir para a compreensão do perfil de adoecimento dessa população a partir dos indicadores de hospitalização, haja vista que a existência de determinantes próprios de cada componente etário pode implicar na existência de tendências distintas.

\section{Metodologia}

Trata-se de um estudo de tendência temporal compreendendo o período de 2000 a 2015, con- siderando todos os municípios brasileiros. As fontes dos dados foram os Sistema de Informação Hospitalar (SIH) e o Sistema de Informação sobre Nascidos Vivos (SINASC), gerenciado pelo Departamento de Informática do Sistema Único de Saúde (DATASUS), vinculado ao Ministério da Saúde.

Nesse estudo, foram incluídas todas as AIH (Autorizações de Internação Hospitalar) relativas aos registros de internação de crianças menores de um ano de idade em hospitais públicos, privados ou filantrópicos que prestavam serviços ao Sistema Único de Saúde. O conjunto de causas de ICSAP tomou como referência os 19 grupos de doenças publicados na portaria n. 221, de 17 de abril de 2008, que definiu a Lista Brasileira de Condições Sensíveis à Atenção Primária ${ }^{11}$.

Foram calculadas as taxas de internações por condições sensíveis à atenção primária à saúde em menores de 1 ano e seus subcomponentes etários, e as taxas de internação pelos principais grupos de causas específicas: gastroenterites infecciosas, pneumonias bacterianas, doenças pulmonares, asma, infecção no rim e trato urinário. Além dessas cinco causas principais, observadas nos menores de 1 ano e no grupo pós-neonatal, também foram calculadas as taxas de internações por sífilis congênita nos neonatos, já que essa foi a principal causa de hospitalização nessa faixa etária. Por se tratar de um estudo com população infantil e pelo fato da sífilis congênita representar 91,2\% de todas as internações do grupo "Doenças relacionadas ao pré-natal e parto", optou-se por denominar esse grupo de "Sífilis congênita e outras infecções congênitas".

Para o cálculo das taxas de internações em cada ano, foi utilizado como numerador a quantidade total de internações de menores de 1 ano e seus subcomponentes etários, e denominador o número de nascidos vivos (NV). Considerouse internações em menores de 1 ano aquelas que ocorreram em bebês com idade inferior a 364 dias de vida. Os subcomponentes etários foram divididos em período neonatal, que correspondia às internações cujos bebês tinham até 27 dias de vida, e período pós-neonatal de 28 a 364 dias.

Foram estimadas as tendências temporais das taxas de internações por causas evitáveis (geral e específicas) em crianças menores de um ano e nos seus subcomponentes etários. $\mathrm{Na}$ análise de tendência temporal, foi utilizado o modelo de análise linear generalizado de Prais-Winsten ${ }^{12}$, considerando como variável de desfecho $(\mathrm{Y})$ as taxas de internação e variável de exposição $(\mathrm{X})$ os anos da série temporal. A adequação do modelo 
de Prais-Winsten foi avaliada pelo Teste de Durbin-Watson ${ }^{13}$.

A utilização do modelo de Prais-Winsten baseou-se na metodologia desenvolvida por Antunes e Waldman ${ }^{14-16}$ que propuseram a transformação logarítmica das taxas de internações, que passam a ser o desfecho utilizado no modelo de regressão. Em seguida, os coeficientes de regressão $(\beta 1)$ e os seus respectivos intervalos de confiança de 95\% (IC95\%), estimados pelo modelo, são transformados em um indicador de variação percentual anual (APC, do inglês Anual Percent Change $)^{15}$. A partir do cálculo da variação percentual anual e dos seus respectivos intervalos de confiança, foi possível classificar o comportamento das taxas em "aumento", "redução" e "estabilidade".

\section{Resultados}

No Brasil, de 2000 a 2015, foram registradas 3.138.540 internações por condições sensíveis a APS em crianças menores de 1 ano, com redução percentual anual de 7,4\% (IC95\%: -10,9; -3,8), e variação das taxas de ICSAP de 72,7/1.000 NV a 48,14/1.000 NV (Gráfico 1). No grupo de neonatos, as taxas de ICSAP apresentaram aumento percentual anual de 6,4\% (IC95\%: 3,1; 9,7), saltando de 4,36/1.000 NV para 7,40/1.000 NV. Já nos pós-neonatos, houve diminuição percentual anual de $8,9 \%$ (IC95\%: -12,5; -5,2) nessas taxas, reduzindo de $68,41 / 1.000 \mathrm{NV}$ para $40,74 / 1.000$ NV (Gráfico 2).
Do total de hospitalizações, as gastroenterites infecciosas $(39,2 \%)$ representaram a principal causa para o conjunto de menores de 1 ano. Em relação aos componentes neonatal e pós-neonatal, foram observadas diferenças quanto às causas mais frequentes em cada grupo, sendo o grupo da sífilis congênita e outras infecções congênitas responsável pela maior proporção de internações em neonatos $(29,5 \%)$, enquanto as gastroenterites foram às de maior magnitude no grupo de crianças do período pós-neonatal (41,1\%) (Tabela 1 ).

Em relação aos grupos de causas específicas de ICSAP em crianças no período neonatal, as taxas de hospitalização por sífilis congênita e outras infecções congênitas apresentaram aumento percentual anual de 24,8\% (IC95\%: 11,0 - 38,5), o maior acréscimo nessa faixa etária. Além desse grupo, também foi observado aumento nas taxas de hospitalização por doenças pulmonares e por infecções do rim e trato urinário. Notou-se estabilidade nas taxas de internações por pneumonias bacterianas e redução de 16\% (IC95\%: $-18,8 ;-13,0)$ nas taxas de hospitalizações por gastroenterites (Tabela 2).

A análise da tendência de ICSAP em crianças do período pós-neonatal demonstrou redução de 22,6\% (IC95\%: -25,6; -19,5) nas taxas de internações por gastroenterites e diminuição, em menor magnitude, nas taxas de hospitalizações por asma. Ainda nesse grupo etário, destaca-se o aumento nas taxas de internações por doenças pulmonares e de infecções no rim e trato urinário e padrão de estabilidade nas taxas de pneumonias bacterianas (Tabela 3).

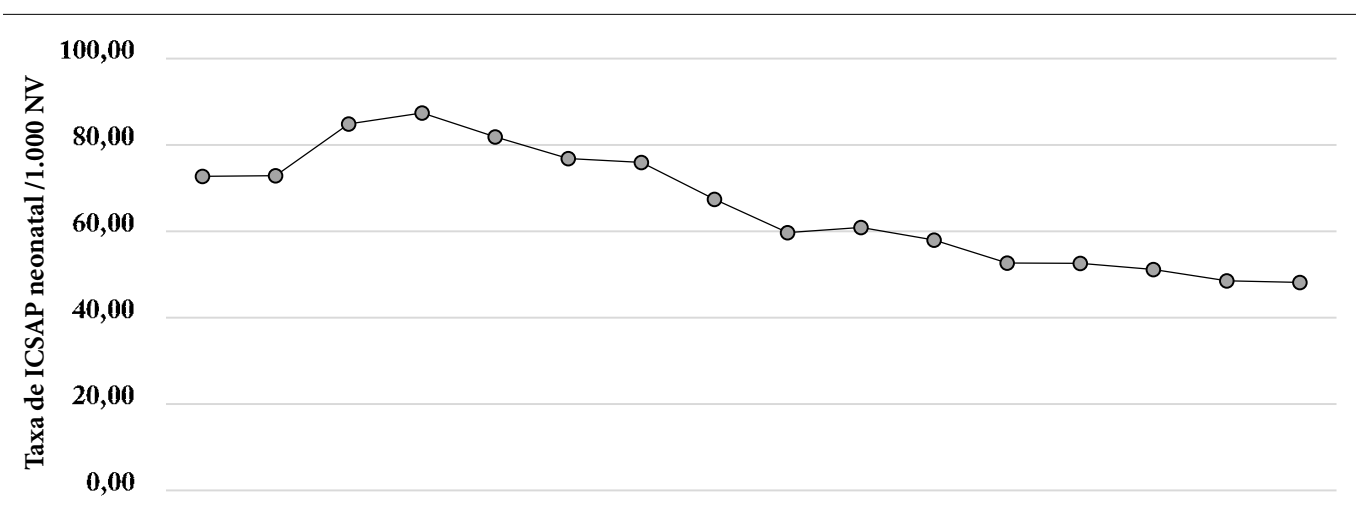

$\begin{array}{llllllllllllllll}2000 & 2001 & 2002 & 2003 & 2004 & 2005 & 2006 & 2007 & 2008 & 2009 & 2010 & 2011 & 2012 & 2013 & 2014 & 2015\end{array}$

Gráfico 1. Taxas de internações por condições sensíveis à atenção primária em menores de 1 ano, Brasil, 20002015. 


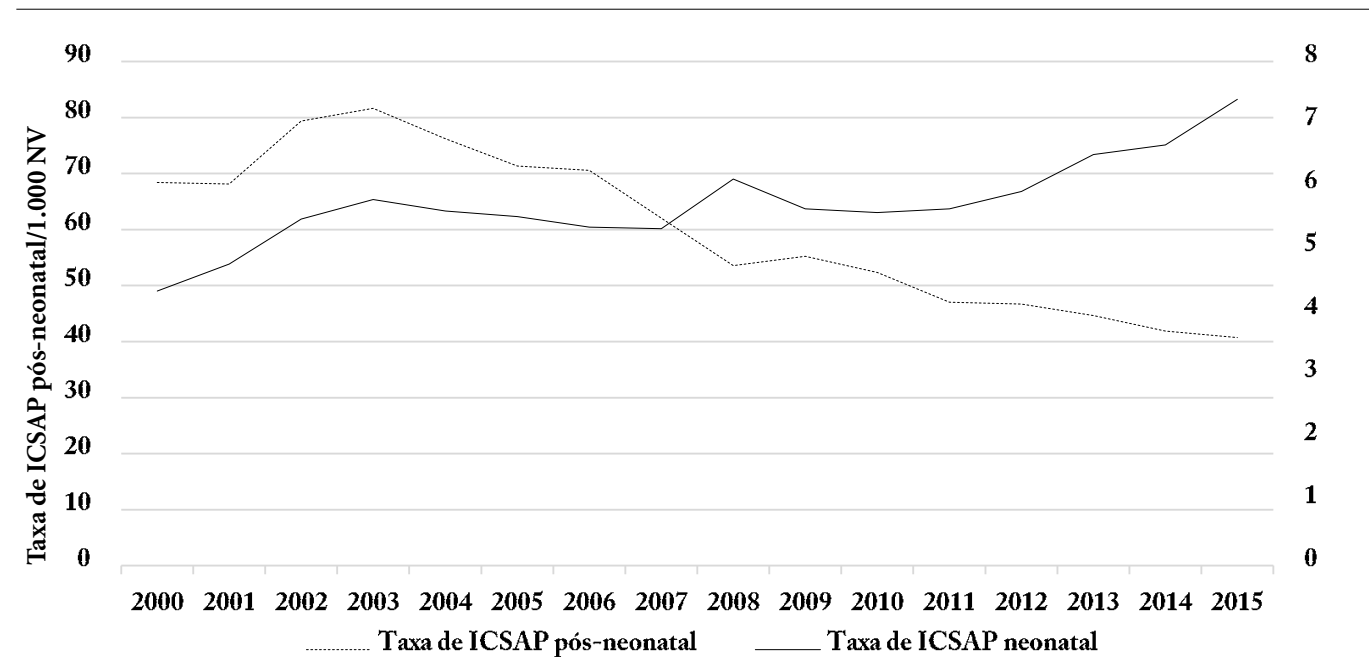

Gráfico 2. Taxas de internações por condições sensíveis à atenção primária em neonatos e pós-neonatos, Brasil, 2000-2015.

Fonte: SIH/SUS e SINASC/SUS (2016).

Tabela 1. Ordenamento das principais causas de internações por condições sensíveis à atenção primária em neonatos, pós-neonatos e menores de 1 ano no Brasil, 2000-2015.

\begin{tabular}{|c|c|c|c|}
\hline Faixa etária & Grupos de causas & $\begin{array}{c}\text { Total de } \\
\text { internações }\end{array}$ & $\begin{array}{c}\text { Proporção de } \\
\text { internações }\end{array}$ \\
\hline Neonato & $1^{\circ}$ Sífilis congênita e outras infecções congênitas & 80.488 & $29,5 \%$ \\
\hline \multirow[t]{5}{*}{ (0-27 dias) } & $2^{\circ}$ Gastroenterites infecciosas & 52.300 & $19,2 \%$ \\
\hline & $3^{\circ}$ Pneumonias bacterianas & 31.432 & $11,5 \%$ \\
\hline & $4^{\circ}$ Doenças pulmonares & 29.930 & $11,0 \%$ \\
\hline & $5^{\circ}$ Infecção no rim e trato urinário & 17.868 & $6,6 \%$ \\
\hline & Todas as ICSAP & 272.443 & - \\
\hline Pós-Neonato & $1^{\circ}$ Gastroenterites infecciosas & 1.176 .965 & $41,1 \%$ \\
\hline \multirow[t]{5}{*}{ (28-364 dias) } & $2^{\circ}$ Pneumonias bacterianas & 479.596 & $16,7 \%$ \\
\hline & 3o Doenças pulmonares & 467.336 & $16,3 \%$ \\
\hline & $4^{\circ}$ Asma & 361.962 & $12,6 \%$ \\
\hline & $5^{\circ}$ Infecção no rim e trato urinário & 114.875 & $4,0 \%$ \\
\hline & Todas as ICSAP & 2.866 .097 & - \\
\hline \multirow[t]{6}{*}{ Menores de 1 ano } & $1^{\circ}$ Gastroenterites infecciosas & 1.229 .265 & $39,2 \%$ \\
\hline & $2^{\circ}$ Pneumonias bacterianas & 510.938 & $16,3 \%$ \\
\hline & $3^{\circ}$ Doenças pulmonares & 497.266 & $15,8 \%$ \\
\hline & $4^{\circ}$ Asma & 373.576 & $11,9 \%$ \\
\hline & $5^{\circ}$ Infecção no rim e trato urinário & 132.743 & $4,2 \%$ \\
\hline & Todas as ICSAP & 3.138 .540 & - \\
\hline
\end{tabular}

Fonte: SIH/SUS (2016). 
Tabela 2. Tendência das taxas de hospitalização em neonatos (x 1.000 nascidos vivos), segundo principais grupos de condições sensíveis à atenção primária, Brasil, 2000-2015.

\begin{tabular}{lccccc}
\hline Ano & $\begin{array}{c}\text { Sífilis congênita e } \\
\text { outras infecções } \\
\text { congênitas }\end{array}$ & $\begin{array}{c}\text { Doenças } \\
\text { pulmonares }\end{array}$ & Gastroenterites & $\begin{array}{c}\text { Infecções do rim e } \\
\text { trato urinário }\end{array}$ & $\begin{array}{c}\text { Pneumonias } \\
\text { bacterianas }\end{array}$ \\
\hline 2000 & 0,78 & 0,43 & 1,46 & 0,18 & 0,30 \\
2001 & 1,23 & 0,45 & 1,58 & 0,21 & 0,24 \\
2002 & 1,14 & 0,45 & 1,69 & 0,27 & 0,76 \\
2003 & 1,45 & 0,46 & 1,55 & 0,28 & 1,00 \\
2004 & 1,46 & 0,53 & 1,32 & 0,26 & 1,00 \\
2005 & 1,48 & 0,50 & 1,28 & 0,30 & 0,91 \\
2006 & 1,52 & 0,51 & 1,17 & 0,30 & 0,91 \\
2007 & 1,42 & 0,60 & 1,03 & 0,35 & 0,89 \\
2008 & 1,30 & 0,74 & 1,21 & 0,49 & 0,76 \\
2009 & 1,26 & 0,70 & 0,96 & 0,54 & 0,76 \\
2010 & 1,44 & 0,67 & 0,92 & 0,51 & 0,63 \\
2011 & 1,66 & 0,80 & 0,77 & 0,48 & 0,55 \\
2012 & 1,96 & 0,79 & 0,76 & 0,48 & 0,47 \\
2013 & 2,47 & 0,85 & 0,71 & 0,47 & 0,55 \\
2014 & 3,03 & 0,75 & 0,57 & 0,47 & 0,41 \\
2015 & 3,69 & 0,91 & 0,49 & 0,48 & 0,42 \\
APC & $24,0 \%$ & $12,6 \%$ & $-16,0 \%$ & $16,5 \%$ & $1,9 \%$ \\
$($ IC95\%) & $(11,0-38,5)$ & $(10,1-15,1)$ & $(-18,8--13,0)$ & $(7,9-25,7)$ & $(-16,7-24,7)$ \\
Tendência & Aumento & Aumento & Redução & Aumento & Estabilidade \\
\hline APC:Aner & & & & \\
\end{tabular}

APC: Anual Percent Change.

Fonte: Sistema de Informação Hospitalar - SIH/SUS.

Tabela 3. Tendência das taxas de hospitalização em crianças no período pós-neonatal (x1.000 nascidos vivos), segundo principais grupos de condições sensíveis à atenção primária, Brasil, 2000-2015.

\begin{tabular}{lccccc}
\hline Ano & Gastroenterites & $\begin{array}{c}\text { Pneumonias } \\
\text { bacterianas }\end{array}$ & $\begin{array}{c}\text { Doenças } \\
\text { pulmonares }\end{array}$ & Asma & $\begin{array}{c}\text { Infecção do rim } \\
\text { e trato urinário }\end{array}$ \\
\hline 2000 & 40,11 & 3,54 & 7,47 & 10,18 & 1,30 \\
2001 & 40,40 & 3,04 & 7,65 & 9,93 & 1,52 \\
2002 & 39,94 & 13,85 & 7,02 & 11,42 & 1,76 \\
2003 & 37,59 & 17,53 & 7,09 & 11,37 & 1,88 \\
2004 & 34,02 & 16,24 & 7,72 & 10,64 & 1,89 \\
2005 & 32,89 & 14,04 & 7,59 & 9,09 & 2,15 \\
2006 & 30,76 & 14,94 & 8,42 & 8,98 & 2,19 \\
2007 & 21,63 & 14,43 & 9,57 & 9,22 & 2,29 \\
2008 & 20,63 & 9,45 & 9,64 & 6,62 & 2,30 \\
2009 & 19,18 & 10,45 & 10,84 & 6,64 & 2,99 \\
2010 & 18,78 & 8,85 & 10,89 & 5,83 & 2,96 \\
2011 & 13,23 & 7,82 & 12,63 & 5,26 & 2,87 \\
2012 & 12,99 & 7,00 & 13,05 & 4,67 & 3,09 \\
2013 & 10,46 & 7,26 & 13,52 & 4,22 & 3,15 \\
2014 & 10,14 & 6,49 & 12,03 & 3,40 & 3,22 \\
2015 & 8,37 & 6,67 & 13,07 & 3,33 & 3,40 \\
APC & $-22,6 \%$ & $3,6 \%$ & $10,9 \%$ & $-17,0 \%$ & $14,9 \%$ \\
(IC95\%) & $(-25,6--19,5)$ & $(-17,9-30,6)$ & $(7,5-14,4)$ & $(-21,6--12,1)$ & $(12,1-17,8)$ \\
Tendência & Redução & Estabilidade & Aumento & Redução & Aumento \\
\hline APC:Anto & & & &
\end{tabular}

APC: Anual Percent Change.

Fonte: Sistema de Informação Hospitalar - SIH/SUS. 


\section{Discussão}

Os resultados deste estudo evidenciaram redução nas taxas de hospitalizações evitáveis pela APS de crianças menores de 1 ano, com perfis de causas e tendências temporais distintas nos grupo neonatal e pós-neonatal, no Brasil. Nos neonatos, foi observada tendência de aumento nas taxas de ICSAP, sendo a sífilis congênita a principal causa. No componente pós-neonatal, a tendência foi de queda nas taxas de internações por condições sensíveis, sendo as gastroenterites infecciosas a causa de maior magnitude. $\mathrm{Na}$ análise das causas específicas de hospitalizações evitáveis, foram identificados diferentes comportamentos dentro de cada componente etário, sendo observado a coexistência de grupos de causas com tendência de redução, enquanto outras aumentaram ou se mantiveram estáveis. Além disso, é preciso ressaltar que, apesar da tendência de redução, as taxas de ICSAP em menores de 1 ano ainda são elevadas, revelando assim a magnitude desse problema.

A tendência de redução nas taxas de ICSAP em crianças menores de 1 ano, em especial no período pós-neonatal, deu-se por conta da diminuição das hospitalizações por gastroenterites, que representaram as causas de maior magnitude nessa faixa etária. Desse modo, é plausível pensar que ações sobre os determinantes das internações por gastroenterites impactaram de maneira significativa as hospitalizações evitáveis no período pós-neonatal. Além disso, essa faixa etária representa a maioria das hospitalizações em menores de 1 ano, de modo que seu comportamento também reflete o esperado nas análises em populações infantis. Logo, pode-se considerar que a redução das taxas de hospitalizações por gastroenterites, especialmente no período neonatal, dada a sua magnitude, influenciaram de modo contundente o comportamento das taxas de ICSAP em menores de 1 ano.

Alguns estudos demonstraram a tendência de redução nas taxas ICSAP na população brasilei$\mathrm{ra}^{4,6}$, mas, em relação às crianças, foi encontrado apenas um estudo que abordou a tendência de internação em todo o território nacional, cuja análise considerou o período de 1999 até $2006^{3}$, e demonstraram resultados semelhantes aos descritos nessa pesquisa.

$\mathrm{Na}$ literatura internacional, estudos apontam que as internações por causas evitáveis em crianças são associadas à um conjunto de determinantes socioeconômicos, sendo observadas maiores taxas desse indicador em áreas de maior vulne- rabilidade $^{17,18}$. Outros estudos nessa temática evidenciaram que a redução destas hospitalizações evitáveis pode ser atribuída à melhoria de determinantes sociais da saúde e ampliação do acesso aos serviços de $\mathrm{APS}^{19}$, que no Brasil se refletem na expansão da Estratégia Saúde da Família (ESF) ${ }^{20}$. A expansão da cobertura desses serviços tem impactado na redução das internações por condições sensíveis em menores de cinco anos ${ }^{21}$, além de colaborar na redução de outros desfechos negativos, como o óbito infantil, o que tem sido atribuído aos aumentos da cobertura de ações de imunização, do número de consultas pré-natal e de puericultura e das ações coletivas em saúde nas áreas cobertas pelas equipes de saúde da família ${ }^{22}$.

As investigações sobre internações neonatais por condições sensíveis à APS ainda são incipientes na literatura científica. A maioria dos estudos que analisaram esse grupo etário investigou as admissões em unidades de terapia intensiva ou a mortalidade desses bebês, o que dificulta as comparações com os achados dessa pesquisa. A tendência de aumento nas taxas de ICSAP nesse grupo etário no Brasil pode ser atribuída a diversos fatores. Cogita-se que a expansão dos serviços do Sistema Único de Saúde (SUS) possa ter ampliado o acesso à assistência hospitalar, suprimido uma demanda por serviços de maior complexidade em lugares antes desassistidos. Entretanto, também é preciso considerar que os problemas na assistência ao pré-natal e parto podem ter influenciado no adoecimento desses bebês, expondo-os a maior risco de internação após o parto e nos primeiros dias de vida ${ }^{23,24}$.

Outro importante achado deste estudo foi a tendência de aumento das taxas de internações por doenças relacionadas ao pré-natal e parto no grupo de crianças do período neonatal. Esse grupo de doenças tem como principal causa a sífilis congênita, morbidade cuja incidência tem aumentado a ponto de ser considerada uma epidemia no Brasil. As causas dessa epidemia têm sido atribuídas ao aumento dos casos de sífilis na população adulta, especialmente, em mulheres, devido à transmissão vertical da doença, relacionado com problemas da assistência pré-natal às mães diagnosticadas com sífilis. Apesar da expansão dos serviços de APS, com ampliação da cobertura de consultas pré-natais, persistem problemas estruturais e organizacionais que comprometem a qualidade destas ações no país ${ }^{25,26}$.

A discussão sobre as ICSAP em neonatos ainda precisa considerar questões que dizem respeito à evitabilidade da internação neste grupo, 
ponderando quais causas que dispensam a hospitalização e aquelas cuja internação é desejável, mesmo estando presentes na lista brasileira de condições evitáveis. Nesses últimos casos, onde se cogita que a internação seja desejável, dada a dificuldade do manejo no âmbito ambulatorial, o aumento nas hospitalizações pode revelar ampliação do acesso aos serviços hospitalares, com potencial efeito na redução da mortalidade.

Um estudo que avaliou o efeito da ESF sobre as internações infantis por pneumonia ${ }^{27}$, doença cujo manejo clínico indica a hospitalização como a melhor opção de tratamento em bebês de até dois meses de vida ${ }^{28}$, concluiu que a expansão na cobertura da Estratégia Saúde da Família esteve associada ao aumento das internações por pneumonia nesses bebês. Esses achados demonstraram que o aumento nas taxas de ICSAP nesse grupo etário representou efeitos positivos, já que o sistema de saúde foi capaz de identificar as necessidades dessa população no nível mais básico e encaminhá-los para serviços de mais alta complexidade ${ }^{27}$.

Além disso, destaca-se como limitação do estudo os problemas inerentes ao uso dos dados secundários do Sistema de Informação Hospitalar, como os erros diagnósticos no preenchimento da causa básica de internação nas Autorizações de Internação Hospitalar. Apesar dessas limitações, é preciso destacar os avanços no aprimoramento desse sistema e na confiabilidade diagnóstica das AIH para a classificação das hospitalizações como condições sensíveis à APS. Aponta-se ainda a utilidade dos dados secundários, de acesso público, para a realização de estudos epidemiológicos de tendência temporal, com abrangência nacional, sem a necessidade de grande aporte financeiro e logístico para a sua execução $0^{29,30}$.

Outro ponto a ser destacado é unicidade da Lista de Brasileira de causas evitáveis de internação, sendo importante a realização de ajustes na sua definição para que se tenha um instrumento capaz de captar as especificidades de determinadas faixas etárias. Dessa forma, a criação de listas específicas, a exemplo da Lista Brasileira de Mortes Evitáveis por Intervenções do Sistema Único de Saúde que considera um grupo de causas para pessoas de 0 a 4 anos e outro para 5 a 74 anos $^{31}$ podem auxiliar na construção de indicadores de ICSAP ainda mais úteis para a avaliação, planejamento e gestão em saúde.

O presente estudo apresentou uma descrição detalhada das ICSAP em menores de 1 ano no Brasil, destacando as tendências e principais causas nos diferentes grupos etários (neonatos e pós-neonatos). A opção por um estudo de caráter descritivo revelou suas potencialidades ao demonstrar, com maior riqueza de detalhes, os distintos comportamentos desse indicador.

As diferenças nas tendências de ICSAP em menores de 1 ano, que podem refletir a influência de determinantes específicos no risco de internar em cada um dos subcomponentes etários e apontam a necessidade de estudá-los separadamente. A compressão dos distintos fatores associados às ICSAP em neonatos e pós-neonatos pode permitir a implantação de intervenções específicas e efetivas em cada uma das faixas etárias, no sentido de produzir redução dessas morbidades e das suas repercussões negativas na vida das crianças e de suas famílias. Nesse sentido, aponta-se a necessidade de realização de outros estudos que investiguem de que modo os programas de proteção social, como o Bolsa Família, e a expansão do acesso à serviços de saúde, especialmente no âmbito da Estratégia Saúde da Família, influenciam as tendências das internações evitáveis em neonatos e pós-neonatos. Além disso, sugere-se ainda a investigação das tendências e perfis de ICSAP em menores de 1, segundo estados e regiões do Brasil, a fim de conhecer os diferentes comportamentos desse indicador nos diversos cenários que compõem esse país.

\section{Colaboradores}

EP Pinto Junior participou da concepção e projeto, análise e interpretação dos dados, redação do artigo. R Aquino participou da concepção e projeto, interpretação dos dados e revisão crítica relevante do conteúdo intelectual do artigo. I Dourado participou da interpretação dos dados e revisão crítica relevante do conteúdo intelectual do artigo. LQ Costa participou da concepção e projeto, interpretação dos dados, redação do artigo. MGC Silva participou da concepção e projeto, interpretação dos dados e revisão crítica relevante do conteúdo intelectual do artigo.

\section{Agradecimentos}

Ao Conselho Nacional de Desenvolvimento Científico e Tecnológico (CNPq), pela concessão da Bolsa de Doutorado ao autor EP Pinto Junior. 


\section{Referências}

1. Billings J, Zeitel L, Lukomnik J, Carey TS, Blank AE, Newman L. Impact of socioeconomic status on hospital use in New York City. Health Aff (Millwood) 1993; 12(1):162-173.

2. Alfradique ME, Bonolo PF, Dourado I, Lima-Costa MF, Macinko J, Mendonça CS, Oliveira VB, Sampaio LFR, De Simoni C, Turci MA. Internações por condições sensíveis à atenção primária: a construção da lista brasileira como ferramenta para medir o desempenho do sistema de saúde (Projeto ICSAP-Brasil). Cad Saúde Pública 2009; 25(6):1337-1349.

3. Moura BLA, Cunha RC, Aquino R, Medina MG, Mota ELA, Macinko J, Dourado I. Principais causas de internação por condições sensíveis à atenção primária no Brasil: uma análise por faixa etária e região. Rev Bras Saúde Matern Infant 2010; 10(Supl. 1):S83-S91.

4. Dourado I, Oliveira VB, Aquino R, Bonolo P, Lima-Costa MF, Medina MG, Mota E, Turci MA, Macinko J. Trends in primary health care-sensitive conditions in Brazil: the role of the Family Health Program (Project ICSAP-Brazil). Med Care 2011; 49(6):577-584.

5. Boing AF, Vicenzi RB, Magajewski F, Boing AC, Moretti-Pires RO, Peres KG, Lindner SR, Peres MA. Reduction of ambulatory care sensitive conditions in Brazil between 1998 and 2009. Rev Saude Publica 2012; 46(2):359-366.

6. Ceccon RF, Meneghel SN, Viecili PRN. Internações por condições sensíveis à atenção primária e ampliação da Saúde da Família no Brasil: um estudo ecológico. Rev Bras Epidemiol 2014; 17(4):968-977.

7. Carvalho SC, Mota E, Dourado I, Aquino R, Teles C, Medina MG. Hospitalizations of children due to primary health care sensitive conditions in Pernambuco State, Northeast Brazil. Cad Saude Publica 2015; 31(4):744-754.

8. Barreto JOM, Nery IS, Costa MSC. Estratégia Saúde da Família e internações hospitalares em menores de 5 anos no Piauí, Brasil. Cad Saude Publica 2012; 28(3):515-526.

9. Prezotto KH, Chaves MMN, Mathias TAF. Hospitalizações sensíveis à atenção primária em crianças, segundo grupos etários e regionais de saúde. Rev Esc Enferm USP 2015; 49(1):44-53.

10. Barros FC, Matijasevich A, Requejo JH, Giugliani E, Maranhão AG, Monteiro CA, Barros AJD, Bustreo F, Merialdi M, Victoria CG. Recent trends in maternal, newborn, and child health in Brazil: progress toward Millennium Development Goals 4 and 5. Am J Public Health 2010; 100(10):1877-1889

11. Brasil. Ministério da Saúde (MS). Secretaria de Atenção à Saúde. Portaria no 221, de 17 de abril de 2008. Define a Lista Brasileira de Condições Sensíveis à Atenção Primária. Diário Oficial da União 2008; 18 abr.

12. Prais SJ, Winsten CB. Trend estimators and serial correlation. Chicago: Cowles Commission; 1954.

13. Durbin J, Watson GS. Testing for serial correlation in least squares regression: I. Biometrika 1950; 37(3-4):409-428.

14. Antunes JLF, Waldman EA. Trends and spatial distribution of deaths of children aged 12-60 months in São Paulo, Brazil, 1980-98. Bull World Health Organ 2002; 80(5):391-398.

15. Antunes JLF, Cardoso MRA. Uso da análise de séries temporais em estudos epidemiológicos. Epidemiol Serv Saúde 2015; 24(3):565-576.

16. Cunha AP, Cruz MM, Torres RMC. Tendência da mortalidade por aids segundo características sociodemográficas no Rio Grande do Sul e em Porto Alegre: 2000-2011. Epidemiol Serv Saúde 2016; 25(3):477-486.
17. Bettenhausen JL, Colvin JD, Berry JG, Puls HT, Markham JL, Plencner LM, et al. Association of Income Inequality With Pediatric Hospitalizations for Ambulatory CareSensitive Conditions. JAMA Pediatr 2017, 171(6):e170322.

18. Roos LL, Dragan R, Schroth RJ. Pediatric ambulatory care sensitive conditions: Birth cohorts and the socio-economic gradient. Can J Public Health 2017; 108(3):e257-e264.

19. Ansari Z. The concept and usefulness of Ambulatory Care Sensitive Conditions as indicators of quality and access to primary health care. Austr J Primary Health 2007; 13(3):91-110.

20. Monahan LJ, Calip GS, Novo PM, Sherstinsky M, Casiano M, Mota E, Dourado I. Impact of the Family Health Program on gastroenteritis in children in Bahia, Northeast Brazil: an analysis of primary care-sensitive conditions. J Epidemiol Glob Health 2013; 3(3):175-185.

21. Rosano A, Loha CA, Falvo R, Van Der Zee J, Ricciardi W, Guasticchi G, Belvis AG. The relationship between avoidable hospitalization and accessibility to primary care: a systematic review. Eur J Public Health 2013; 23(3):356-360.

22. Aquino R, Oliveira NF, Barreto ML. Impact of the family health program on infant mortality in Brazilian municipalities. Am J Public Health 2009; 99(1):87-93.

23. Viellas EF, Domingues RMSM, Dias MAB, Gama SGN, Theme Filha M, Costa JV, Bastos MH, Leal MC. Assistência pré-natal no Brasil. Cad Saúde Pública 2014; 30 Suppl:85-100.

24. Nunes JT, Gomes KRO, Rodrigues MTP, Mascarenhas MDM. Qualidade da assistência pré-natal no Brasil: revisão de artigos publicados de 2005 a 2015. Cad Saúde Colet 2016; 24(2):252-261.

25. Luz LA. Avaliação da qualidade da atenção pré-natal na Estratégia de Saúde da Família no Brasil [dissertação]. Salvador: Instituto de Saúde Coletiva UFBA; 2016.

26. Cooper JM, Michelow IC, Wozniak PS, Sánchez PJ. Em tempo: a persistência da sífilis congênita no Brasil Mais avanços são necessários! Rev Paulista Pediatr 2016; 34(3):251-253.

27. Pamponet ML. O efeito do Programa Saúde da Família nas internações hospitalares por pneumonias em crianças menores de cinco anos no Brasil [dissertação]. Salvador: Instituto de Saúde Coletiva UFBA; 2011.

28. Diretrizes brasileiras em pneumonia adquirida na comunidade em pediatria - 2007. J Bras Pneumol 2007; 33(Supl. 1):s31-s50.

29. Abaid, R, Nedel F, Alcayaga E. Condições sensíveis à atenção primária: confiabilidade diagnóstica em Santa Cruz do Sul, RS. Rev Epidemiol Controle Infecção 2014; 4(3):208-214.

30. Machado JP, Martins M, Leite IC. Qualidade das bases de dados hospitalares no Brasil: alguns elementos. Rev Bras Epidemiol 2016; 19(3):567-581.

31. Malta DC, Duarte EC, Almeida MF, Dias MAS, Morais Neto OL, Moura L, Ferraz W, Souza MFM. Lista de causas de mortes evitáveis por intervenções do Sistema Único de Saúde do Brasil. Epidemiol Serv Saúde 2007; 16(4):233244.

Artigo apresentado em 27/06/2018

Aprovado em 26/10/2018

Versão final apresentada em 28/10/2018 